\title{
Une pédagogie éclectique au service des élèves qui ont le plus besoin de l'école
}

Et si, une fois encore, l'enseignement spécialisé ouvrait la voie à un renouvellement de la réflexion pédagogique? S'il parvenait, avant l'enseignement ordinaire, à réaliser la synthèse des avancées les plus significatives des pratiques d'enseignement au service des apprentissages des élèves les plus fragiles, ceux qui ont le plus besoin de l'école pour apprendre. À lire ce numéro de la Nouvelle revue de l'adaptation et de la scolarisation, on peut raisonnablement le penser.

\section{L'enfer est pavé de bonnes intentions}

Dans l'enseignement spécialisé comme dans l'enseignement ordinaire, la pédagogie est à un tournant. Une période s'achève, celle de la rénovation pédagogique amorcée au début des années 70, et une autre commence, portée par un discours, qualifié tantôt de réactionnaire tantôt de pragmatique, qui revalorise des pratiques jugées plus traditionnelles. L'erreur serait de ne voir dans ce retour de balancier que la simple traduction sur le plan pédagogique de l'idéologie politique dominante. Cela conduirait à laisser le champ libre aux pratiques les plus rétrogrades alors qu'au contraire un nouvel équilibre pédagogique progressiste, visant une réduction des inégalités scolaires, pourrait être recherché.

Pour y parvenir, il importe de ne pas s'arc-bouter sur des principes sans s'interroger sur l'impact réel des pratiques que ceux-ci ont générées. En pédagogie aussi, l'enfer est pavé de bonnes intentions; on peut vouloir le bien des élèves des milieux populaires et obtenir l'effet inverse. C'est l'une des vertus de la recherche en éducation que de le rappeler dans le droit fil des travaux de sociologie des années 60. À la suite de Bourdieu ${ }^{1}$, de nombreux chercheurs ont montré les effets néfastes de pédagogies « invisibles » ${ }^{2}$, celles qui sont peu intelligibles aux élèves et à leurs parents, peu explicites dans leur mise en œuvre et lacunaires quant aux savoirs enseignés : trop de compétences requises par les tâches scolaires ne sont pas assez exercées à l'école et sont laissées à la charge de l'éducation hors l'école, ce qui contribue à renforcer les inégalités. Bref une pédagogie insuffisamment explicite, progressive et structurée ne permet pas de réduire les inégalités scolaires, voire les accroit. ${ }^{3}$

\section{Sortir d'une pensée binaire}

Les débats contemporains les plus intéressants, si l'on met de côté les caricatures qui font les choux gras des polémistes de la rentrée des classes, invitent à dresser un bilan critique des pratiques pédagogiques dont les effets ont été contraires aux intentions qui avaient présidé à leur élaboration. C'est ce que nous nous efforçons, nous aussi, de faire ${ }^{4}$. Ces débats sont nourris par des sociologues, des psychologues, des didacticiens et des pédagogues que l'on ne peut suspecter de vouloir restaurer je ne sais quel ordre établi ou

\footnotetext{
${ }^{1}$ Bourdieu , 1996

2 Bernstein, 1995, 2007; Bonnery, 2007.

${ }^{3}$ Rosenshine, 1989 ; Terrail, 2004 ; Bissonnette, Richard et Gauthier, 2005.

${ }^{4}$ Goigoux, 2006 (a et b), 2010 ; Nonnon et Goigoux, 2007.
} 
favoriser l'élitisme, fut-il républicain ${ }^{5}$. Leur but est plutôt d'essayer de comprendre pourquoi, parmi les pays occidentaux, la France fait partie de ceux dans lesquels le déterminisme social pèse le plus fort sur les performances scolaires des élèves ${ }^{6}$ et de chercher à identifier les caractéristiques des pédagogies qui pourraient contribuer à une réduction des inégalités sociales. L'objectif n'est pas de nier les avancées des innovations réalisées depuis quarante ans mais de contribuer à rectifier les malentendus ou les erreurs qu'elles ont générés?

Cet objectif est difficile à atteindre car la pensée politique contemporaine en éducation est excessivement binaire. Les chercheurs sont sommés de choisir leur camp entre « républicains » et "pédagogues », entre défenseurs des savoirs et défenseurs des enfants, entre savoirs et compétences, entre pédagogie et didactique ou bien encore entre " pédagogie centrée sur l'enseignement » et "pédagogie centrée sur l'élève », le dernier avatar du dualisme pédagogique! Bref, on nous demande de penser noir ou blanc, quand nos investigations portent sur une vaste palette de dégradés de gris.

Nous nous y refusons. C'est pourquoi nous voudrions esquisser ici les contours d'une pédagogie que nous qualifierons d'éclectique c'est-à-dire, au sens donné par Littré, « qui admet ce que chaque système paraît offrir de bon ». Nous voudrions promouvoir une attitude consistant à choisir dans plusieurs systèmes les éléments qui paraissent les plus sages et les plus pertinents pour constituer un système cohérent et, si possible, plus efficient que ceux qui sont aujourd'hui proposés aux enseignants pour orienter leurs pratiques professionnelles. Nous voudrions essayer de transposer modestement dans notre champ une part de la réflexion philosophique de Diderot $^{8}$ pour défendre l'éclectisme pédagogique en l'opposant au dogmatisme, qui vire souvent au sectarisme, et au syncrétisme qui s'efforce de concilier l'inconciliable, de juxtaposer des théories contradictoires.

L'entreprise est risquée, nous le savons, et le danger est grand de ne pas éviter le second écueil, celui du syncrétisme, qui est le plus redoutable même si les pamphlétaires de tous bords accaparent les médias en jouant avec la peur du premier, le sectarisme. L'entreprise est néanmoins nécessaire si l'on veut aider les enseignants à œuvrer dans un sens favorable aux apprentissages des élèves le plus en difficulté sans se sentir coupables de renier leur histoire professionnelle ou de renoncer à leurs valeurs.

\section{Un bilan critique}

Cette histoire professionnelle a valorisé les pédagogies de «projet » ou le recours aux situations d'apprentissages « naturelles » ou « complexes », aujourd'hui contestées au nom du principe de l'organisation hiérarchique des habiletés'. Les thèses pédagogiques inspirées par l'Éducation nouvelle (improprement qualifiées de pédagogies constructivistes alors que le constructivisme est une théorie psychologique et non pédagogique) sont aujourd'hui contestées lorsqu'elles conduisent à une fréquentation trop aléatoire, trop peu hiérarchisée, trop espacée et trop peu fréquente des savoirs visés par l'enseignement. Leurs

5 Par exemple Danièle Manesse (2003) dans le domaine de l'enseignement du français, Denis Butlen ou Marie-Lise Pelletier (2002, 2004) en didactique des mathématiques, Marcel Crahay (2008) en psychologie de l'éducation, Jean-Pierre Terrail (2002), d'Élisabeth Bautier et Jean-Yves Rochex (1997), Patrick Rayou (2009) ou Stéphane Bonnery (2007) en sociologie de l'éducation ou bien encore celle de Pascal Bressoux (2001, 2007) en sciences de l'éducation, pour n'en citer que quelques uns.

${ }^{6}$ OCDE, 2006 ; DEP, 2008 ; OCDE, 2010.

${ }^{7}$ Charlot, Bautier et Rochex, 1993 ; Bautier et Goigoux, 2004.

${ }^{8}$ Article Éclectisme de l'Encyclopédie

9 Péladeau, Forget et Gagné, 2010. 
défauts de planification sont pointés du doigt ${ }^{10}$, ainsi que l'habillage des situations pseudoconcrètes qui conduisent les élèves à s'égarer sur de fausses pistes cognitives ${ }^{11}$ et à perdre les bénéfices qu'offrent une sélection, une présentation et une organisation rigoureuses de situations didactiques "artificielles »: matériel épuré, stabilité des formats des tâches scolaires, découpage des contenus, rythme de progression et gradation des difficultés, etc.

Les élèves les plus vulnérables ont besoin d'une pédagogie qui respecte une « loi d'optimum " proposant un habile dosage entre une forte part de connu (répétitions intratâches) et une moindre, mais néanmoins consistante, part de nouveauté (variabilités intraet inter-tâches). Lorsque le guidage didactique est réduit à son minimum, lorsqu'on omet l'apprentissage d'habiletés préalables à la résolution de problèmes complexes ou l'entraînement explicite d'habiletés élémentaires, les écarts entre les meilleurs élèves et ceux ayant des difficultés augmentent, alors qu'ils se réduisent dans le cas d'une hiérarchisation des habiletés et d'un guidage progressif des apprentissages au sein de situations rigoureusement construites dans ce but ${ }^{12}$.

Trois éléments apparaissent dès lors comme décisifs : 1) la définition précise des objectifs et l'évaluation de ce qui a été effectivement enseigné, 2) la pratique régulière de l'évaluation formative (renforcement intrinsèque), 3) l'enseignement préalable des compétences requises pour un nouvel apprentissage. Ce sont des conditions qui ne sont pas l'apanage des pédagogies de maîtrise ${ }^{13}$ : des recherches récentes ont montré que certaines écoles Freinet par exemple ${ }^{14}$ pouvaient obtenir des réussites comparables lorsqu'elles combinaient phases de tâtonnement et planifications rigoureuses favorisant un enrôlement des élèves dans des tâches pertinentes sur le plan cognitif.

\section{L'éclectisme pédagogique}

La pédagogie que nous défendons, et qui semble gagner du terrain ${ }^{15}$, cherche à concilier les acquis des pédagogies actives avec les exigences des pédagogies explicites et structurées. Elle combine des phases d'enseignement déclaratif (exposition de règles, de procédures ou de notions), des phases de résolution guidée sous la tutelle étroite de l'enseignant et des phases de tâtonnement, d'exploration ou de découverte (recours à des situations-problèmes) tout en accordant le plus grand soin aux phases d'entrainement, d'exercice ou de jeu qui favorisent la mémorisation des notions et l'automatisation des procédures.

Contrairement à une idée répandue, cette pédagogie n'est pas en contradiction avec les programmes en vigueur à l'école primaire dont le préambule de 2008 stipule : « Chacun s'accorde aujourd'hui sur l'utilité d'un apprentissage structuré des automatismes et des savoir-faire instrumentaux comme sur celle du recours à des situations d'exploration, de découverte, ou de réflexion sur des problèmes à résoudre. [...] L'accès au sens et l'acquisition des automatismes ne sont pas antinomiques : c'est aux enseignants de varier les approches et les méthodes pour lier ces deux composantes de tout apprentissage. »

Tout nous encourage par conséquent à poursuivre notre travail dans le sens défini en collaboration avec Sylvie Cèbe depuis une quinzaine d'années ${ }^{16}$. Les principes qui sont les nôtres sous-tendent les outils didactiques que nous élaborons. À titre d'exemple, en

\footnotetext{
${ }^{10}$ Ausubel, 2000.

11 Bautier et Rayou, 2007.

${ }^{12}$ Crahay et Dutrevis, 2010.

13 Boom, 1979.

${ }^{14}$ Reuter, 2008.

${ }^{15}$ Crahay et Dutrevis, 2010 ; Nonnon et Goigoux, 2007.

${ }^{16}$ Cèbe et Goigoux, 1999.
} 
voici quelques-uns rendus concrets dans Lector et Lectrix ${ }^{17}$, un manuel pour l'enseignement de la lecture avec des élèves de l'enseignement secondaire en grande difficulté.

\section{- Planifier un enseignement explicite}

Apprendre à comprendre exige, pour de nombreux élèves, un enseignement explicite de stratégies : la simple pratique réitérée de la lecture ne suffit pas pour que tous connaissent les procédures et les stratégies qu'utilisent les lecteurs confirmés, encore moins à leur apprendre à les utiliser à bon escient. C'est pourquoi nous proposons une organisation pédagogique en plusieurs étapes: 1) annonce et explication des apprentissages visés, 2) présentation des problèmes à résoudre et des procédures à employer (utiles à la compréhension), 3) pratique dirigée par l'enseignant (application de ces procédures dans le traitement de plusieurs tâches différentes), 4) pratique autonome, 5) synthèse collective (analyse et prise de conscience des effets de leur mise en œuvre sur la qualité de la compréhension), 6) révision régulière.

\section{- Favoriser la clarté cognitive}

Notre objectif est d'offrir aux élèves la plus grande clarté cognitive possible touchant l'activité de lecture d'une part et le dispositif d'enseignement d'autre part. C'est pourquoi nous mettons tout en œuvre pour qu'ils sachent ce qu'ils sont en train de faire et ce qu'on cherche à leur faire apprendre. Dans nos outils didactiques, chaque séance commence par le rappel de ce qui a été précédemment appris. Le professeur annonce et justifie l'objectif de la séance puis, au fil de la séance, il explique le but de toutes les tâches. Il invite toujours les élèves à s'interroger a priori sur les procédures à utiliser et à justifier leur choix. Au terme de chaque activité, les élèves sont amenés à mettre en relation les procédures employées avec le résultat obtenu. Chaque séquence s'achève par une synthèse qui fait le point sur ce qui vient d'être appris et, parfois, la rédaction d'une fiche mémoire.

Pour montrer aux élèves que les apprentissages réalisés améliorent réellement la qualité de leur compréhension, nous leur proposons, à la fin de chaque séquence, d'effectuer plusieurs tâches qui requièrent la mobilisation des procédures enseignées. Avant de les laisser travailler seuls, le professeur les incite à analyser ces tâches de transfert et à s'interroger sur les stratégies qu'il convient d'appliquer pour les réaliser efficacement.

\section{- Favoriser l'engagement des élèves dans les activités}

Compte tenu de leur histoire scolaire, souvent émaillée d'échecs et d'incompréhensions, les plus faibles lecteurs ont besoin d'être remobilisés pour accepter de poursuivre l'apprentissage de la lecture. Certains d'entre eux ont même renoncé à croire que, dans ce domaine au moins, ils pourraient encore progresser. Avec Lector et Lectrix, leur engagement durable dans l'apprentissage résulte plus de l'accroissement de leur sentiment de compétence en lecture que de l'intérêt porté aux histoires lues. Il repose aussi sur le constat que les efforts consentis se soldent bien par une amélioration de leur compréhension et donc des réussites en actes. Celles-ci les confortent aussi dans leur capacité à progresser.

Pour permettre cette remobilisation, nous sommes attentifs à ne pas submerger les élèves par des demandes qui s'avèrent hors de leur portée et à ne pas les laisser, trop vite,

${ }^{17}$ Cèbe et Goigoux, 2009. 
trop seuls. C'est pourquoi nous mettons tout en œuvre pour que la prise de risque soit modérée et progressive.

Nous commençons toutes les activités par un travail collectif qui offre l'avantage de protéger les élèves les plus faibles de l'échec individuel et du jugement de leurs camarades (ou de leur professeur!). Rien, en effet, ne les oblige à s'exposer publiquement s'ils ne se sentent pas suffisamment prêts ou sûrs de leur réponse. Ce mode de travail permet aussi de multiplier les tâches dont la résolution publique sera immédiatement commentée, dispositif qui permet aussi d'augmenter les occasions de faire comprendre aux élèves ce qu'on attend d'eux. Le guidage de l'enseignant permet enfin de leur faire découvrir les procédures qu'ils ignorent et qu'ils ne peuvent inventer seuls. Le fait que les élèves ne bénéficient pas, à ce moment-là, d'un feed-back personnalisé ne nous inquiète pas : l'objectif est ailleurs, dans le repérage de l'apprentissage à réaliser.

Nous protégeons aussi les élèves le plus en difficulté en retenant des modalités pédagogiques qui évitent de prendre la classe à témoin de leur incompétence. Nous proposons, par exemple, des tâches originales qui consistent à juger de la qualité des réponses d'autres élèves fictifs. Dispensés d'avoir à défendre leur production ou à sauver la face devant leurs camarades, tous les élèves peuvent s'interroger sur les procédures qui ont conduit d'autres élèves à proposer ces réponses.

\section{- Assurer une attention conjointe}

Trop souvent, les modalités de travail offertes aux élèves ne facilitent pas le travail intellectuel attendu. Ainsi, par exemple, il n'est pas rare que le travail collectif commence sans que les moins performants aient pu consacrer à la lecture du texte le temps nécessaire. Submergés par leurs difficultés, ils restent spectateurs des échanges entre leurs camarades et le professeur et n'osent pas prendre la parole pour ne pas prêter le flanc aux moqueries ou montrer qu'ils ont besoin d'aide.

Par conséquent, nous préconisons de laisser le temps à tous les élèves de réaliser les tâches demandées avant d'organiser une phase de mise en commun. Pour permettre à tous de participer à cet échange collectif, nous leur demandons souvent de répondre à une ou deux consignes, pour eux-mêmes et par écrit (sur leur cahier de brouillon). «Pour euxmêmes » parce qu'il s'agit de solutions privées que personne, ni l'enseignant, ni leurs camarades, n'aura le droit de lire; "par écrit», pour garder une trace de leur raisonnement. Cette modalité pédagogique a pour but d'inciter chaque élève à réfléchir, seul, au problème posé sans que son raisonnement soit court-circuité par les plus rapides ou les plus performants. C'est pour la même raison que les mises en commun sur les différentes représentations d'un même texte ou la correction d'un exercice réalisé individuellement empruntent toujours le même scénario pédagogique que nous appelons " 1 puis 2 puis tous »: le professeur interroge un premier élève qui donne sa réponse et la justifie en ayant recours au texte. Il sollicite ensuite à un autre élève qui doit indiquer et justifier son accord ou son désaccord avec le point de vue de son camarade puis il demande au reste de la classe s'il y a une contestation du résultat ainsi obtenu.

Dans la plupart des activités collectives, nous proposons d'afficher le texte étudié au tableau pour favoriser une attention partagée (à l'aide des posters proposés, d'un rétro ou d'un vidéo projecteur) : ce dispositif facilite le guidage du professeur et le repérage, par les élèves du passage du texte qui fait l'objet de la discussion. Il permet aussi d'éviter de perdre du temps à faire rechercher individuellement l'information dont on parle ou, au contraire, de masquer certaines informations. Bref, il permet de réguler plus aisément les interactions et de diminuer les contraintes qui pèsent sur l'activité, celle du professeur et 
celle des élèves. L'affichage collectif est aussi un auxiliaire précieux pour contrôler l'accès au texte et bloquer l'impulsivité des élèves qui se précipitent trop souvent sur leur feuille avant d'avoir clarifié le but de leur lecture ou de leur relecture.

\section{- Réduire la complexité des tâches}

La régulation de la compréhension en lecture, par exemple la capacité à ralentir, s'arrêter ou revenir en arrière dans un texte pour dépasser une incompréhension passagère, exige une prise de conscience de ses propres stratégies de lecture. Or cette dernière n'est possible que si le lecteur a pu réussir, éventuellement avec l'aide de l'enseignant, à comprendre le texte proposé.

Par conséquent, nous évitons de multiplier les sources de complexité (linguistiques, textuelles, culturelles) dans une même tâche et nous demandons parfois aux enseignants de prendre en charge une part des traitements dévolus normalement aux élèves (par exemple déchiffrer) pour que ceux-ci puissent entièrement se consacrer aux opérations qui sont les cibles de l'enseignement. Nous procédons aussi à de nombreuses lectures à haute voix dont une des fonctions est de permettre à tous, même les moins habiles déchiffreurs, de bien connaître le texte afin de pouvoir participer à la réflexion collective.

De manière générale, nous proposons des tâches que tous les élèves peuvent réussir pour qu'ils puissent ensuite réfléchir aux procédures qu'ils ont utilisées; nous leur demandons souvent de raisonner sur des tâches déjà effectuées par d'autres élèves (fictifs) en leur demandant de s'interroger sur les procédures que ces derniers ont mises en œuvre. Bref, nous multiplions les dispositifs d'aide à la compréhension puis nous en faisons l'objet des prises de conscience (métacognition).

\section{- Stabiliser les formats}

Face à un problème nouveau, les élèves les moins performants ont tendance à solliciter immédiatement l'aide de leur professeur, le plus souvent parce qu'ils se sentent impuissants à le résoudre sans lui. Ils sont donc, plus que les autres, sensibles aux changements qui, loin de les motiver, les inquiètent. En leur proposant sans cesse des tâches et des consignes nouvelles, on les prive de pouvoir exercer un contrôle sur leur propre fonctionnement et on exacerbe leur dépendance à l'adulte. C'est pourquoi nous optons pour des scénarios stables, tant pour le déroulement des séances que pour le choix des exercices ou les modalités d'aide et de guidage. Cette stabilisation des différents formats vise à permettre aux élèves de réaliser une expérimentation effective, prolongée et diversifiée des différentes tâches et activités. Elle leur permet aussi d'anticiper les procédures à mobiliser et de prendre une part de plus en plus importante dans le contrôle de leur activité. Elle rend le monde scolaire plus prévisible : se sentir suffisamment en sécurité est pour nous une condition nécessaire à la réflexion. Enfin, elle permet aux élèves de devenir véritablement « experts » d'un texte ou d'une procédure. La prise de conscience de cette expertise, même locale, renforce un sentiment de compétence qui entraîne à son tour de nouveaux progrès.

\section{- Répéter sans lasser}

Les opérations intellectuelles qui sous-tendent une compréhension efficace sont multiples et complexes. Elles demandent du temps et de la réitération pour être apprises et utilisées par les élèves. Toutefois, les enseignants le savent bien, la répétition à l'identique d'une même activité (même menée plus lentement et en plus petit groupe) ne suffit pas à garantir le succès d'un apprentissage. Il faut donc consacrer beaucoup de temps à exercer 
une même procédure dans de nombreuses tâches dont les buts varient pour solliciter les élèves dans des registres cognitifs différents. Il faut aussi faire varier les modes d'organisation sociale : amener les élèves à travailler seuls, à deux, en groupe ou en classe entière.

\section{- Enseigner les procédures requises par les tâches scolaires, notamment d'évaluation}

Nous ne voulons négliger aucune des habiletés qui fondent la réussite en lecture. À l'école, en effet, il ne suffit pas de comprendre les textes, il faut aussi pouvoir montrer qu'on les a compris en réussissant des épreuves d'évaluation dont les modalités de résolution sont rarement enseignées. Dans les évaluations nationales, par exemple, on demande aux élèves de choisir le meilleur résumé parmi quatre propositions ou de remplir un tableau à double entrée qui associe les noms des personnages d'un récit avec leurs désignations anaphoriques sans que la bonne manière d'effectuer ces tâches ait fait l'objet d'un enseignement explicite. Cette élucidation est également absente des pratiques pédagogiques pour les questions ouvertes qui exigent de rédiger une réponse portant sur l'implicite du texte ou pour les tâches qui demandent de justifier son point de vue. Il n'est donc pas rare que des élèves échouent à certaines épreuves alors même qu'ils ont compris le texte.

C'est pourquoi nous apprenons aux élèves à réaliser les tâches le plus couramment proposées à l'école en consacrant du temps à leur analyse, à l'explicitation des procédures requises pour les réussir et leur prise de conscience. Notre première cible est le questionnaire dont l'école primaire use (et abuse parfois) sans apprendre aux élèves à le traiter de manière stratégique. La réussite du plus grand nombre masque souvent, aux yeux des enseignants, le désarroi des élèves qui comprennent les réponses données par leurs camarades mais ignorent comment ils auraient pu les trouver seuls. C'est pourquoi nous proposons plusieurs séances consacrées à l'élucidation et la prise de conscience des contrats évaluatifs implicites.

Mis en œuvre en S.E.G.P.A depuis plusieurs années, ces principes semblent porter leurs fruits même si nous devons reconnaître que nous n'avons pas procédé à leur évaluation rigoureuse. On ne peut à la fois être partie et juge. Seuls de multiples observations de classes et de nombreux témoignages de satisfaction des enseignants nous servent aujourd'hui de validation et d'encouragement. 


\section{Bibliographie}

Ausubel, D.P. (2000). The acquisition and retention of knowledge: a cognitive view. Boston: Kluwer Academy. Bautier É. et Rochex J.-Y. (1997). Apprendre : des malentendus qui font la différence. In J.-P. Terrail (ed.), La scolarisation de la France. Critique de l'état des lieux. Paris : La Dispute.

Bautier, E. et Goigoux, R. (2004). Difficultés d'apprentissage, processus de secondarisation et pratiques enseignantes : une hypothèse relationnelle, Revue française de pédagogie, 148, 89-100.

Bautier, E. et Rayou, P. (2009). Les inégalités d'apprentissage. PUF.

Bernstein B. (1975). Classes et pédagogies : visibles et invisibles. Paris : CERI-OCDE.

Bernstein B. (2007). Pédagogie, contrôle symbolique et identité. Laval : Presses Universitaires de Laval.

Bissonnette, S. Richard, M.et Gauthier, C. (2005). Interventions pédagogiques efficaces et réussite scolaire des élèves provenant de milieux défavorisés. Note de synthèse. Revue Française de Pédagogie, $\mathrm{n}^{\circ} 150$.

Bloom, B.S. (1979). Caractéristiques individuelles et apprentissages scolaires. Bruxelles : Labor. Paris : Nathan.

Bonnery, S. (2007). Comprendre l'échec scolaire. Élèves en difficultés et dispositifs pédagogiques. Paris : La Dispute.

Bourdieu, P. (1996). L'école conservatrice. Les inégalités devant l'école et devant la culture. Revue française de sociologie. $\mathrm{Vol} 7, \mathrm{n}^{\circ} 3,325-347$.

Bressoux, P. (2001). Réflexions sur l'effet-maître et l'étude des pratiques enseignantes. Les Dossiers des Sciences de l'Education, 5, 35-52.

Bressoux, P. (2007). Qu'est-ce qui caractérise l'enseignant efficace? In V. Dupriez et G. Chapelle (Eds.), Enseigner (pp. 95-106). Paris: PUF.

Butlen, D., Peltier-Barbier, M-L., Pézard, M. (2002). Nommés en REP, comment font-ils ? Pratiques de professeurs d'école enseignant les mathématiques en REP : contradictions et cohérence, Revue française de pédagogie, $\mathrm{n}^{\circ} 140,41-52$.

Cèbe, S. et Goigoux, R. (1999). L'influence des pratiques d'enseignement sur les apprentissages des élèves en difficulté. Cahiers Alfred Binet (renommés Recherches et Educations), 661/4, 49-68.

Cèbe, S. et Goigoux, R. (2008). Apprendre aux élèves à besoins éducatifs particuliers à mieux comprendre les textes lus : un défi pour les enseignants spécialisés. Revue de Neuropsychologie du Développement et des Apprentissages, $\mathrm{n}^{\circ} 92, \mathrm{~T} 2,74-81$.

Cèbe, S. et Goigoux, R. (2009). Lectrix et lector, Apprendre à comprendre les textes narratifs. Paris : Retz

Charlot, B. (1997). Du rapport au savoir. Paris: Anthropos.

Charlot, B., Bautier, E. et Rochex, J.-Y. (1992). École et savoir dans les banlieues... et ailleurs. Paris: Armand Colin.

Crahay, M. (1996). Peut-on lutter contre l'échec scolaire? Bruxelles: De Boeck.

Crahay, M. (2002). Enseigner, entre réussir et comprendre. Théories implicites de l'éducation et pensée des enseignants experts. Essai de recadrage socio-constructiviste. In J. Donnay et M. Bru (Eds.), Recherches, pratiques et savoirs en éducation (pp. 107-132). Bruxelles: De Boeck.

Crahay, M. et Dutrevis, M. (2010). Psychologie des apprentissages scolaires. De Boeck.

Goigoux, R. (2000). Apprentissage et enseignement de la lecture dans l'enseignement adapté. Suresnes : CNEFEI. Synthèse consultable sur http://www.bienlire.education.fr/04media/documents/synthesegoigoux.pdf

Goigoux, R. (2003). Enseigner la compréhension : l'importance de l'auto-régulation, In M. Fayol et D. Gaonac'h (Eds.): Aider les élèves à comprendre: du texte au multimédia (pp. 182-204), Paris : Hachette.

Goigoux, R. (2006a). Apprentissage et enseignement de la lecture auprès d'élèves en grande difficulté. In Centre Alain Savary (Ed.), Apprendre et enseigner en « milieux difficiles » (pp. 85 - 94), Lyon : INRP.

Goigoux, R. (2006b). Erratum - Rectifier les erreurs sur l'apprentissage de la lecture, Les cahiers pédagogiques, 242, 53-56. 
Goigoux, R. (2010). Quelle expertise pédagogique? Les cahiers pédagogiques, 480, 34-35.

Kakpo, S. (2009). Travail hors la classe : familles populaires, familles hyperpartenaires ? In Diversité, VilleEcole-Intégration, 156, 98-103.

Manesse, D. (2003). Le français en classes difficiles. Lyon : INRP.

Nonnon, E. et Goigoux, R. (Dir.) (2006). Surmonter les ratés de l'apprentissage de la lecture à l'école et au collège. Repères, $\mathrm{n}^{\circ} 35$.

Péladeau, N., Forget, J. et Gagné, F. (2010). Le rôle du transfert des apprentissages dans l'acquisition des habiletés simples et complexes. In Crahay, M. et Dutrevis, M. (2010) : Psychologie des apprentissages scolaires (pp. 47-63). De Boeck.

Peltier-Barbier, M -L. (Dir.) (2004). Dur d'enseigner en ZEP. Grenoble : Editions La pensée sauvage.

Reuter,Y. (dir.) (2008). Une école Freinet. Fonctionnements et effets d'une pédagogie alternative en milieu populaire. Paris : L'Harmattan.

Rosenshine, B. (1986). Vers un enseignement efficace des matières structurées. Un modèle d'action inspiré par le bilan des recherches processus-produit. In M. Crahay et D. Lafontaine (Eds.), L'art et la science de l'enseignement (pp. 81-96). Liège: Labor.

Terrail, J.P. (2002). De L'inégalité scolaire, La Dispute.

Terrail, J.P. (2004). Ecole, l'enjeu démocratique, La Dispute. 